\title{
Deep Learning-Based Workflow for Analyzing Helium Bubbles in Transmission Electron Microscopy Images
}

\author{
Chun Yin Wong ${ }^{1}$, Xing Wang ${ }^{2}$, Zhe Fan ${ }^{3}$, Karren More ${ }^{4}$, Sergei Kalinin ${ }^{5}$ and Maxim Ziatdinov ${ }^{6}$
}

${ }^{1}$ University of Tennessee, Knoxville, Tennessee, United States, ${ }^{2}$ The Pennsylvania State University, United States, ${ }^{3}$ Lamar University, United States, ${ }^{4}$ Oak Ridge National Laboratory, United States, ${ }^{5}$ Center for Nanophase Materials Sciences, Oak Ridge National Laboratory, Oak Ridge, Tennessee, United States, ${ }^{6}$ Computational Sciences and Engineering Division, Oak Ridge National Laboratory, Oak Ridge, Tennessee, United States

Optimization of structural materials for nuclear energy purposes necessitates understanding the fundamental mechanisms beyond the helium-induced damage in the structural materials [1]. The morphology of emergent microstructures is readily available via imaging; however, analyzing transmission electron microscopy (TEM) images is time-consuming and often difficult manually or using traditional computational tools. Here we demonstrate the seamless integration of the deep learning algorithms with the physics-based descriptors implemented via traditional computer vision techniques can significantly accelerate the process and provide new insights into the images.

The use of deep convolutional neural networks has long been demonstrated in real-world image and video semantic segmentation, such as in autonomous vehicles [2]. Lately, deep convolutional neural network (DCNN) semantic segmentation has also been employed in microscopy experiments in the biological science and materials science fields to automatically identify features in an image [3]. While DCNNs have been shown to be capable of performing image segmentation, domain knowledge is important in giving physical meaning to the segmented labels. Thus traditional computer vision techniques based on the physics of the formation of the helium bubbles were incorporated to obtain statistics from the labels.

The proposed use of deep learning-based semantic segmentation is part of an overall drive to automate workflows in microscopy experiments. Through the use of deep learning, significant time-savings were gained which accelerated the process of scientific discovery. Furthermore, the use of traditional computer vision techniques as a post-processing step brought to light the importance of incorporating domain knowledge in the application of deep learning in the microscopy field.

Previously, $\mathrm{NiFe}_{\mathrm{x}}$-based single-phase concentrated solid solution alloy samples with different alloy compositions were implanted with $200 \mathrm{keV}$ helium ions to simulate the effects of helium embrittlement in nuclear reactor structural materials [4]. 42 TEM images were acquired, of which ten were labeled and used for the training of the DCNN. The fully-convolutional DCNN had a U-net architecture and ensemble training was used in which twelve models with different weight initializations were trained. Image segmentation was performed on the twelve trained networks individually and the results were averaged. The labeled bubbles were then located and sized using the Laplacian of Gaussian (LoG) and Hough transform separately, and the identified coordinates and radii were concatenated using density-based spatial clustering of applications with noise (DBSCAN). The training and classification workflows are illustrated in Fig. 1. The results of the classification were compared with those manually labeled by three different researchers to measure the performance of the proposed framework.

The performance of the DCNN-based framework was robust. The DCNN ensemble achieved an intersectionover-union (IoU) of 0.85. This was higher than those of the individual neural networks. The identified bubbles are shown in Fig. 2. Upon analyzing the segmented images using the traditional computer vision techniques outlined above, individual bubbles were identified with an IoU of 0.80 . Overlapping bubbles were effectively 
identified separately. Images from other materials systems were also analyzed and the proposed framework was able to identify gas bubbles in such systems effectively even without retraining the network on images of the relevant materials systems.

The demonstration of a successful DCNN-based pipeline not only allowed the same paradigm to be utilized by different experiments to accelerate the process but also brought promise to automating the entire workflow of microscopy experiments - from automatic control of the microscope based on real-time analysis of the image captured, to the data analysis and the generation of new insights from the data acquired.

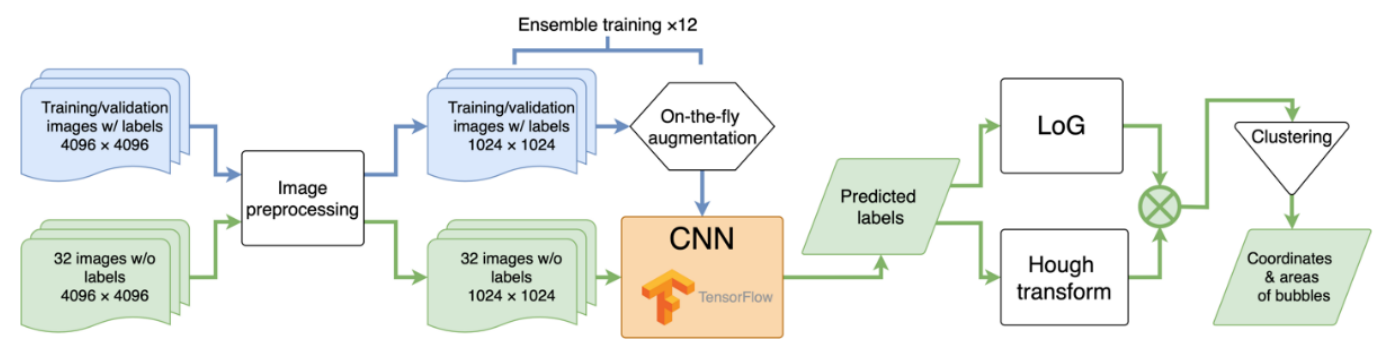

Figure 1. Flowchart of the DCNN-based framework. The blue arrows follow the workflow of the ensemble training process and the green arrows follow the workflow of the classification process.
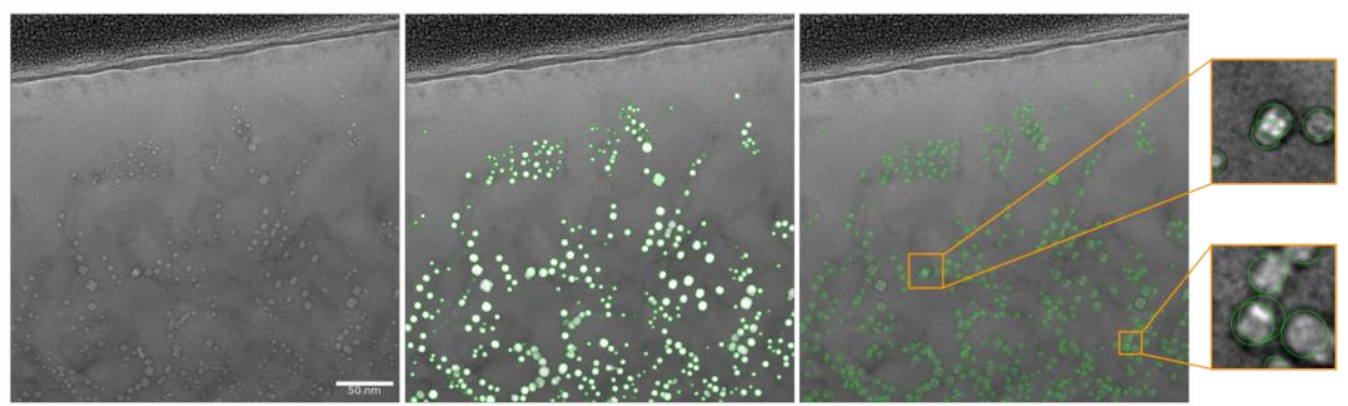

Figure 2. Helium bubbles that were identified by the framework. The insets show examples of overlapping bubbles that were identified as separate bubbles by the framework.

\section{References}

[1] K. Morishita, R. Sugano, and B. D. Wirth, "MD and KMC modeling of the growth and shrinkage mechanisms of helium-vacancy clusters in Fe," Journal of Nuclear Materials, vol. 323, no. 2, pp. 243-250, Dec. 2003, doi: 10.1016/j.jnucmat.2003.08.019.

[2] M. Cordts et al., "The Cityscapes Dataset for Semantic Urban Scene Understanding," in 2016 IEEE Conference on Computer Vision and Pattern Recognition (CVPR), Las Vegas, NV, USA, Jun. 2016, pp. 32133223, doi: 10.1109/CVPR.2016.350.

[3] O. Ronneberger, P. Fischer, and T. Brox, "U-Net: Convolutional Networks for Biomedical Image Segmentation," in Medical Image Computing and Computer-Assisted Intervention - MICCAI 2015, Cham, 2015, pp. 234-241, doi: 10.1007/978-3-319-24574-4_28.

[4] X. Wang et al., "Effects of Fe concentration on helium bubble formation in NiFex single-phase concentrated solid solution alloys," Materialia, vol. 5, p. 100183, Mar. 2019, doi: 10.1016/j.mtla.2018.100183. 